\title{
Simulation of Push-pull Multi-output Quasi-resonant Converter
}

\author{
T.Anitha ${ }^{1}$, S.Arulselvi ${ }^{2}$ \\ ${ }^{I}$ (Assistant Professor, Department of Electronics and Instrumentation Engg., Annamalai University ,India) \\ ${ }_{2}^{2}$ (Professor, Department, of Electronics and Instrumentation Engg., Annamalai University ,India)
}

\begin{abstract}
Power supply voltages in digital systems have been reduced considerably in recent years and often digital components requiring different voltages are present in the same board. This has increased the demand for multiple output power distribution systems with tight load regulation. In this paper, a detailed analysis and design of a multi-output push-pull zero voltage switching (ZVS) quasi resonant converter (QRC) has been carried out. Also simulation of multi-output push-pull zero voltage switching (ZVS) quasi resonant converter $(Q R C)$ has been carried out using Matlab/SIMULINK software. The proposed converter is suitable for aerospace applications.
\end{abstract}

Keywords: $d c-d c$ converters, push-pull converter, quasi-resonant converters, Zero voltage switching.

\section{INTRODUCTION}

Multi-output PWM dc-dc converters are widely used in communications, aerospace and computer systems, as they are usually more compact and less expensive than a collection of single-output converters. Fig.1 shows the schematic of single power converter with multiple outputs, e.g. push-pull converter with multiple secondary windings.

In aerospace applications the allowable size and weight are highly restricted to accommodate greater payload. In the effort to increase the power density of power supplies, the switching frequency is pushed to high values which, in PWM converter realizations, normally lead to considerable power loss, high switching stresses, reduced reliability, and acoustic noise. Another significant drawback of the switch-mode operation is the EMI produced due to large di/dt and dv/dt. To overcome these short comings, new families of QRCs are introduced [1]-[2].Few research papers have been presented about the design and implementation of push-pull hard switched and Quasi-resonant converters[3]-[5].Push-pull converters is mainly used for medium and high power applications compared to half-bridge and full-bridge converter. It has wide duty ratio control and it can be used for multi-output applications with low output and input ripple current.

This paper presents the design of multi-output push-pull ZVS-QRC. The proposed method reduces the switching losses and size of the converter. It can be used as high-density power supply for aero-space applications. All simulation works are carried out using MATLAB/SIMULINK software.
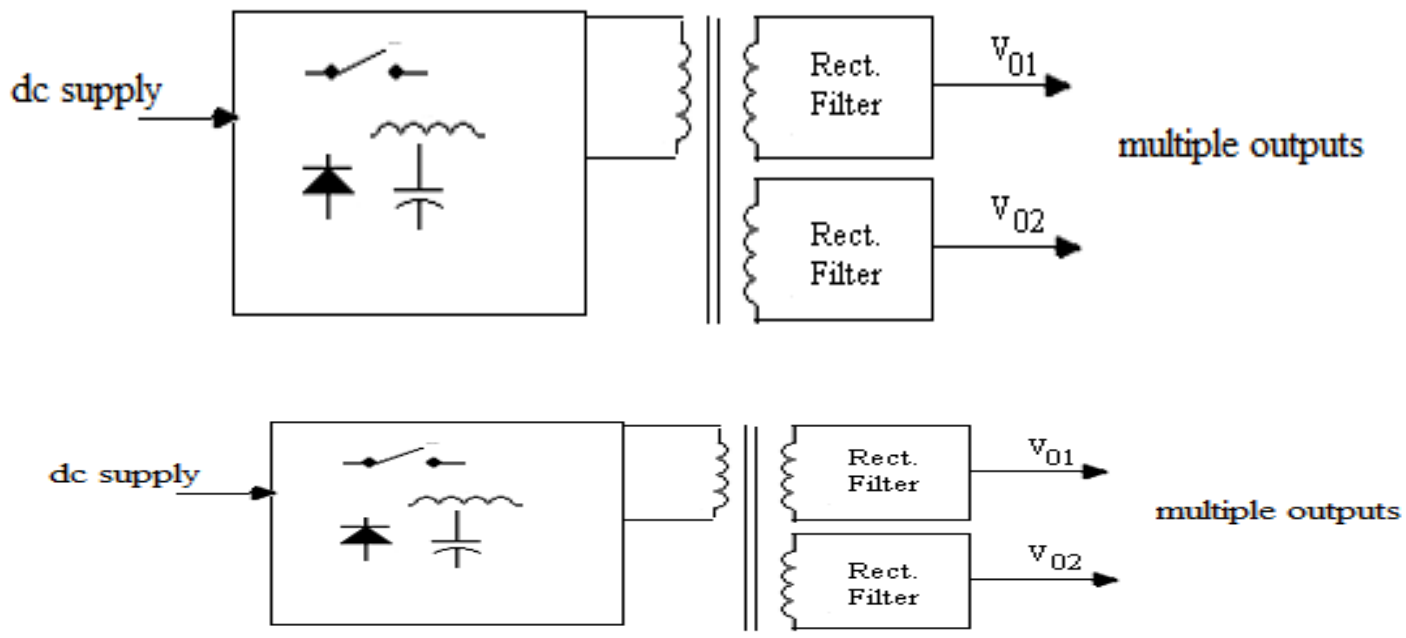

Figure 1 Multiple outputs using single power converter.

II. Analysis Of Multi-OutPut Push-Pull Zvs QRC

The proposed push-pull topology is basically two forward converters connected in anti-phase. An additional two resonant tanks are added in this topology compared to PWM push-pull converter. The resonant 
tank shapes the switch voltage so that the active switches $\left(S_{1}\right.$ and $\left.S_{2}\right)$ are turned on at ZVS. Hence the turn-on losses of the switch will be reduced considerably compared to PWM converters. Both primary and secondary windings are arranged in a center-tapped configuration. The push-pull converter operates in two quadrants (I and III) of the B-H curve, see-sawing back and forth as the each primary is activated. This allows the push-pull converter to deliver twice the maximum power than that of a forward converter. This makes the proposed converter effective for medium and high power applications. The different modes of operation are explained in the following section.
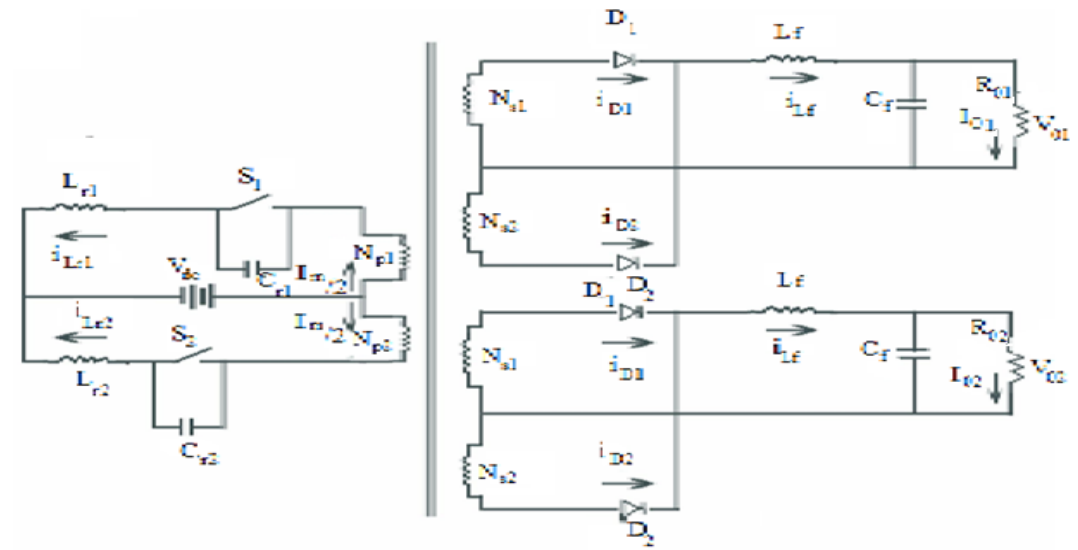

Figure 2 Circuit diagram of multi-output push-pull ZVS-QRC.
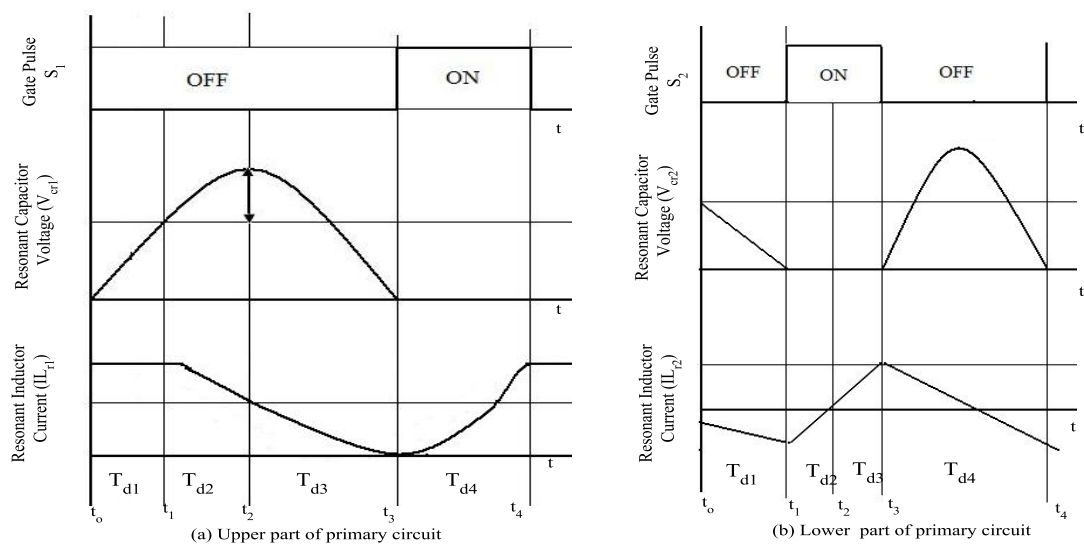

Figure 3 Theoretical resonant waveforms of push-pull ZVS-QRC.

To analyze its steady-state behavior, the following assumptions are made

1. The output filter $C_{f}$ and the load $R_{01}, R_{02}$ are a constant sink of current $\mathrm{I}_{\mathrm{o} 1}$ and $\mathrm{I}_{\mathrm{o} 2}$ respectively.

2. Semiconductor switches are ideal, i.e. no forward voltage drop in the on-state, leakage current in the off state and no time delays at both.

3. Reactive current in the elements are ideal.

4. Magnetizing inductance is larger than resonating inductance

The following parameters are defined

1. Characteristic impedance

2. Resonant angülar frequency

$$
Z_{0}=\sqrt{\left(L_{r} / C_{r}\right)}
$$

$$
\omega_{0}=\frac{1}{\sqrt{\left(L_{r} * C_{r}\right)}}
$$

3. Resonant frequency

$$
f_{0}=\omega_{0} / 2 \pi
$$

4. Normalized Load Resistance 


$$
R=R_{L} / Z_{0}
$$

5. The primary current through primary of transformer $\left(\mathrm{L}_{\mathrm{m}}\right)$ is

$$
I_{L r}=I_{m}
$$

\subsection{MODES OF OPERATION}

The switch $S_{1}$ and $S_{2}$ are alternatively power their respective windings. The resonating capacitors $C_{r 1}$ and $C_{r 2}$ and resonating inductors $L_{r 1}$ and $L_{r 2}$ are used to form the resonant tank. The secondary voltage of the transformer is rectified by fast recovery diodes $\left(\mathrm{D}_{1}\right.$ and $\left.\mathrm{D}_{2}\right)$ and filtered to produce a steady ripple free output voltage $\mathrm{V}_{0}$. Lower and upper part of primary winding circuit is called here afterwards as upper and lower.

\subsubsection{MODE 1: $\left(t_{0}, t_{1}\right)$}

UPPER: The switch $S_{1}$ is opened for the beginning of a new cycle at $t$ equal to $t_{0}$. The current flows through resonant capacitor $\left(C_{r 1}\right)$ and resonant inductor $\left(L_{r 1}\right)$. The capacitor voltage rises linearly from 0 to $2 \mathrm{~V}_{\mathrm{dc}}$. The capacitor voltage is given by the equation,

$$
v_{c r 1}(t)=I_{m} t / 2 C_{r}
$$

When the resonant capacitor voltage reaches $2 \mathrm{~V}_{\mathrm{dc}}$ at $\mathrm{t}=\mathrm{t}_{1}$. The duration of this interval $\mathrm{T}_{\mathrm{d} 1}$ is given by

$$
T_{d 1}=4 C_{r} V_{d c} / I_{m}
$$

LOWER: The switch $\mathrm{S}_{2}$ opened in the previous cycle is still continued. The capacitor voltage $\mathrm{v}_{\mathrm{cr} 2}$ in the lower part decreases linearly from $2 \mathrm{~V}_{\mathrm{dc}}$ to zero. The equations for $\mathrm{v}_{\mathrm{cr} 2}$ and $\mathrm{i}_{\mathrm{Lr} 2}$ are given as

$$
\begin{gathered}
v_{c r 2}(t)=2 V_{d c}-\left(\frac{I_{m}}{2}\right) Z_{o} \sin \omega_{o} t \\
i_{L r 2}=-I_{m} \cos \omega_{0} t
\end{gathered}
$$

2.1.2 MODE 2: $\left(t_{1}, t_{2}\right)$

UPPER: The series resonant $\mathrm{L}_{\mathrm{r} 1}$ and $\mathrm{C}_{\mathrm{r} 1}$ forms a resonant circuit. The state equations are

With initial conditions

$$
\begin{aligned}
& d v_{c r 1} / d t=i_{L r 1}(t) / C_{r 1} \\
& d i_{L r 1} / d t=\left(2 V_{d c}-v_{c r 1}(t)\right) / L_{r 1}
\end{aligned}
$$

$$
\text { and } \quad \begin{aligned}
v_{c r 1}(0) & =2 V_{d c} \\
i_{L r 1}(0) & =I_{m} / 2
\end{aligned}
$$

The solutions for the above equations are given by

$$
\begin{aligned}
& v_{c r 1}(t)=2 V_{d c}+\left(I_{m} / 2\right) Z_{o} \sin \omega_{o} t \\
& i_{L r 1}(t)=\left(I_{m} / 2\right) \cos \omega_{0} t
\end{aligned}
$$

At $\mathrm{t}$ equal to $\mathrm{t}_{2}, i_{L r 1}$ reaches zero value and $v_{c r 1}$ reaches its peak value as shown in fig, $3 \mathrm{a}$. They are given by

$$
v_{c r 1}\left(t_{2}\right)=2 V_{d c}+\left(I_{m} / 2\right) Z_{0}
$$

The duration of the mode $T_{d 2}$ is given by

$$
T_{d 2}=t_{2}-t_{1}=\pi / \omega_{0}
$$

LOWER: The switch $S_{2}$ is turned on when $V_{\mathrm{cr} 2}$ become zero at $t=t_{1}$, to achieve zero voltage condition. During this stage the current $\mathrm{i}_{\mathrm{Lr} 2}$ increases linearly and reaches the value $I_{m} / 2$ at $\mathrm{t}=\mathrm{t}_{2}$. The corresponding state equation for $i_{L r 2}$ is given by

The solution of above equation is given by

$$
d i_{L r 2} / d t=2 V_{d c} / L_{r 2}
$$

$$
i_{L r 2}=\left(I_{m} / 2\right) \cos \omega_{0} t
$$

2.1.3 MODE 3: $\left(t_{2}, t_{3}\right)$

UPPER: The equations determining this stage are

and

$$
d i_{L r 1} / d t=\left(2 V_{d c}-v_{c r 1}(t)\right) / L_{r 1}
$$

$$
d v_{c r 2} / d t=i_{L r 1}(t) / C_{r 1}
$$


With initial conditions

$$
\begin{aligned}
& v_{c r 1}(0)=2 V_{d c} \\
& i_{L r 1}(0)=-I_{m} / 2
\end{aligned}
$$

The solution for the equations are given by

$$
\begin{aligned}
& i_{L r 1}(t)=-\left(I_{m} / 2\right) \cos \omega_{o} t \\
& v_{C r 1}(t)=2 V_{d c}-\left(I_{m} / 2\right) \sin \omega_{o} t
\end{aligned}
$$

This stage terminates when $V_{c r 1}(t)$ becomes zero. The duration of this stage is given by

Where

$$
\begin{aligned}
& T_{d 3}=t_{4}-t_{3} \\
& T_{d 3}=\left(1 / \omega_{0}\right) \sin ^{-1}\left[V_{d c} /\left(I_{m} Z_{0}\right)\right] \\
& T_{d 3}=(\alpha-\pi) / \omega_{0}
\end{aligned}
$$

The inductor current is given by

LOWER: $\mathrm{v}_{\mathrm{cr} 2}$ is zero and

$$
\alpha=\pi+\left(\sin ^{-1}\left[4 V_{d c} / I_{m} Z_{0}\right]\right)
$$

$$
i_{L r 1}=\left(I_{m} / 2\right) \cos \alpha
$$

With initial conditions

$$
d i_{L r 2} / d t=\left(2 V_{d c}-v_{c r 2}(t)\right) / L_{r 2}
$$

The solution for above equation is

$$
i_{L r 2}(0)=I_{m} / 2
$$

$$
i_{L r 2}(t)=\left(I_{m} / 2\right) \cos \left(\omega_{0} t\right)
$$

\subsubsection{MODE 4: $\left(t_{3}, t_{4}\right)$}

UPPER: The switch $S_{1}$ is turned on when $v_{\mathrm{crl}}$ become zero to achieve zero voltage condition. During this mode the current $i_{\text {Lr1 }}$ increases linearly and reaches the value $I_{m} / 2$ at $t$ equal to $t_{4}$. The corresponding state equation is

With initial condition

$$
d i_{L r 1} / d t=2 V_{d c} / L_{r 1}
$$

$$
i_{L r 1}(0)=\left(I_{m} / 2\right) \cos \alpha
$$

The inductor current $i_{L r 1}(\mathrm{t})$ is given by the equation

$$
i_{L r 1}(t)=\left(\left(2 V_{d c} * t\right) / L_{r}\right)+\left(\left(I_{m} / 2\right) \cos \alpha\right)
$$

This mode terminates when inductor current become $I_{m}$. The duration of this stage is given by the equation

$$
\begin{aligned}
& T_{d 4}=t_{4}-t_{3} \\
& T_{d 4}=\left[I_{m} Z_{0} / 4 V_{d c} \omega_{0}\right](1-\cos \alpha)
\end{aligned}
$$

LOWER: The switch $\mathrm{S}_{2}$ is opened at $\mathrm{t}=\mathrm{t}_{4}$. The current flows through resonant capacitor $\left(C_{r 2}\right)$ and resonant inductor $\mathrm{L}_{\mathrm{r} 2}$. The capacitor voltage rise s linearly from zero to $2 V_{d c}$ and it is governed by equation

$$
v_{c r 2}(t)=\left(I_{m} t\right) / 2 C_{r 1}
$$

At the end of this mode, a new mode cycle starts.

\subsection{Theoretical resonant waveforms}

The theoretical resonant waveforms of the proposed converter for one switching cycle are shown in fig.3. Whenever there is a change in switching device from one state to another, the circuit configuration changes. Each configuration is referred to as mode. In this $\mathrm{QRC}$, four different stages are identified for each half of the switching cycle.

\subsection{Design}

The design parameters are given for a multi-output push-pull ZVS-QRC with following specifications: 
- Input voltage

- Output voltage of secondary1

- $\quad$ Output Voltage of secondary2

- Resonant frequency

- Switching frequency

- Turns ratio between primary and secondary

- The characteristic impedance

$$
\begin{aligned}
& V_{d c}=40 \mathrm{~V} \\
& V_{01}=5 \mathrm{~V} \\
& V_{02}=12 \mathrm{~V} \\
& f_{0}=165 \mathrm{kHz} \\
& f_{s}=50 \mathrm{kHz} \\
& n_{1}=N_{p 1} / N_{s 1}=0.125 \\
& n_{2}=N_{p 2} / N_{s 2}=0.3 \\
& Z_{0}=\operatorname{sqrt}\left(L_{r} / C_{r}\right)
\end{aligned}
$$

- The resonant capacitors are assumed to be equal

$$
\begin{aligned}
& C_{r}=C_{r 1}=C_{r 2}=0.047 \text { microF } \\
& L_{r}=L_{r 1}=L_{r 2}=0.16 \mathrm{mH}
\end{aligned}
$$

- The resonant inductors are assumed to be equal

\section{OPEN LOOP STUDIES}

In this section the open loop simulation of multi-output push-pull ZVS-QRC circuit is realized with parameters obtained from the design. The simulated resonant capacitor voltage and output voltage of the converter are discussed in this section. The performance of the converter is studied for different load variations.

The converter is simulated using Matlab/SIMULINK software in open loop with design parameters. The SIMULNK model of the circuit is shown in figure 4. The gating pulses applied to switch $S_{1}$ and $S_{2}$ are shown in fig. 5. It is observed that the switch $S_{1}$ and $S_{2}$ are turned on, when the resonant capacitor voltage becomes zero to assure ZVS condition. The waveform resembles the theoretical waveforms as shown in fig 3.

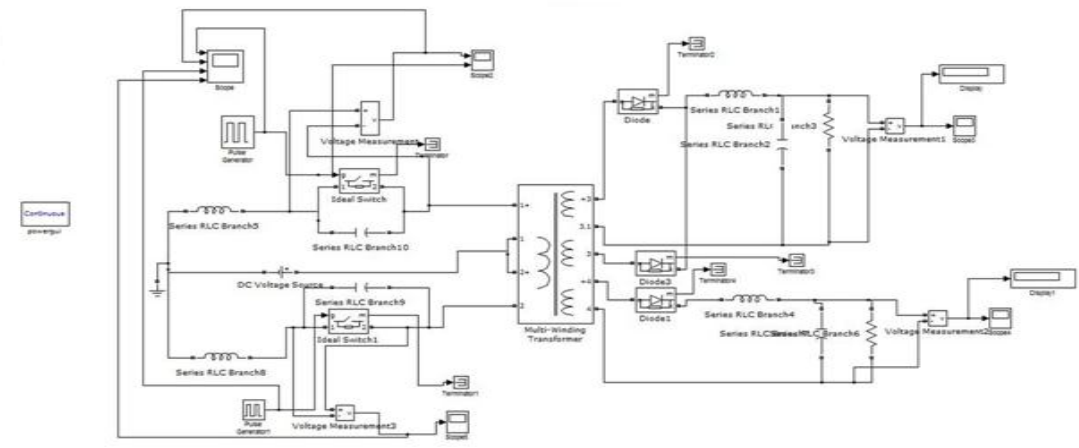

Figure 4 Simulink Model Diagram for the Multi Output Push-Pull ZVS-QRC

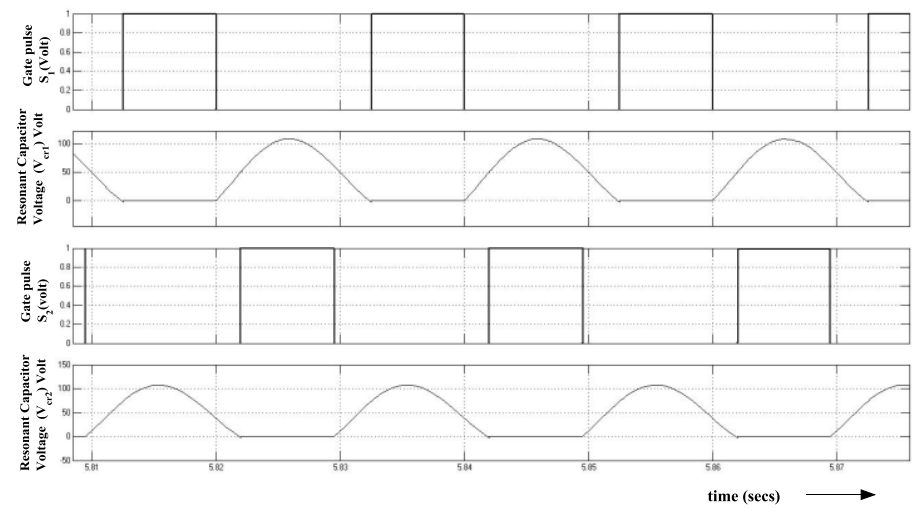

Figure 5 Simulated resonant waveforms for upper part and lower part of primary. 


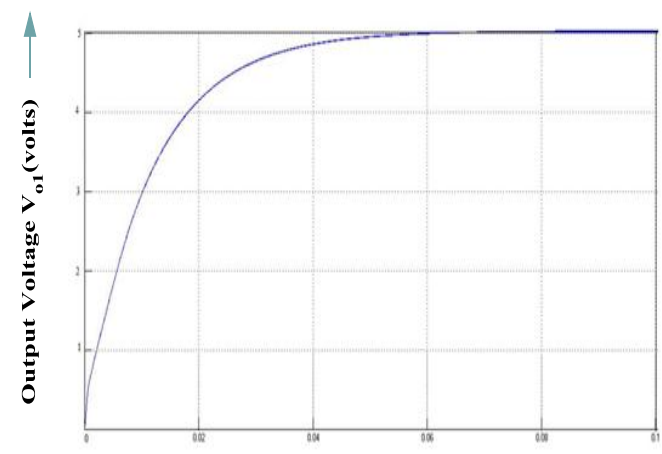

time(secs)

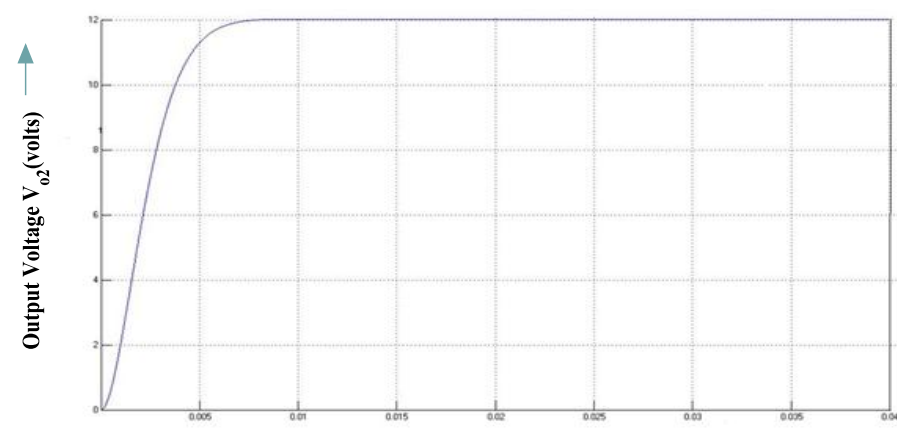

time(secs)

Figure 6 Simulated open loop output waveforms of the secondary $1 V_{01}$ and secondary $2 V_{02}$ (volts).

\section{CONCLUSION}

Theoretical analysis and design procedure of a new buck type push-pull ZVS-QRC was presented. The converter rated at $100 \mathrm{~W}$, operating at switching frequency of $50 \mathrm{kHZ}$ has been simulated using Matlab/SIMULINK software. Operating principle and theoretical analysis are confirmed by simulation results. The converter can also be implemented in hardware which will give matching results as that of simulated results. Hence this is suitable for high-density, medium power requirement, for example in aerospace applications.

\section{REFERENCES}

[1] Liu K.H. and LEE F.C.Y., Zero-voltage Switching Technique in DC-Dc Converters, IEEE Specialists Conference Record, 1986,pp.58-70.

[2] I.Barbi, J.C.Bolacell,D.C.Martins and F.B.Libano, Buck Quasi-Resonant Converter operating at Constant Frequency:Analysis, Design and Experimantation,IEEE,pp.873-880.

[3] Wildon C.P., de Aragao Filho,I.Barbi, A Comparison Between Two Current-Fed Push-Pull DC-DC Converters-Analysis,Design and Experimentation, IEEE, 1996, pp.313-320.

[4] Grover V., T.Bascope and I.Barbi, Isolated Flyback-Current-Fed Push-Pull Converter For Power Factor Correction,IEEE,1996,pp.1184-1190.

[5] B.Swaminathan and V.Ramanarayanan, A Novel Resonant Transition Push-Pull DC-DC Converter,J.Indian Inst Sci,Nov.Dec.2004,pp.217-232 